\title{
MicroRNA-6498-5p Inhibits Nosema bombycis Proliferation by Downregulating BmPLPP2 in Bombyx mori
}

\author{
Congwu Hu ${ }^{1}$, Zhanqi Dong ${ }^{1,2}{ }^{\mathbb{D}}$, Boyuan Deng ${ }^{1}$, Qin $\mathrm{Wu}^{1}{ }^{1}$, Peng Chen ${ }^{1,2}$, Cheng Lu ${ }^{1,2, *}$ and Minhui Pan ${ }^{1,2, *}$ \\ 1 State Key Laboratory of Silkworm Genome Biology, Southwest University, Chongqing 400716, China; \\ 18013127742@126.com (C.H.); zqdong@swu.edu.cn (Z.D.); dengboyuan99@163.com (B.D.); \\ wuuqin@163.com (Q.W.); pjchen@swu.edu.cn (P.C.) \\ 2 Key Laboratory of Sericultural Biology and Genetic Breeding, Ministry of Agriculture and Rural Affairs, \\ Southwest University, Chongqing 400716, China \\ * $\quad$ Correspondence: lucheng@swu.edu.cn (C.L.); cs_pmh@swu.edu.cn (M.P.); Tel.: +86-023-68250346 (C.L.); \\ +86-023-68250076 (M.P.); Fax: +86-023-68251128 (C.L. \& M.P.)
}

check for updates

Citation: Hu, C.; Dong, Z.; Deng, B.; Wu, Q.; Chen, P.; Lu, C.; Pan, M. MicroRNA-6498-5p Inhibits Nosema bombycis Proliferation by Downregulating BmPLPP2 in Bombyx mori. J. Fungi 2021, 7, 1051. https:// doi.org/10.3390/jof7121051

Academic Editor: Mathieu Nacher

Received: 14 November 2021

Accepted: 6 December 2021

Published: 8 December 2021

Publisher's Note: MDPI stays neutral with regard to jurisdictional claims in published maps and institutional affiliations.

Copyright: (C) 2021 by the authors. Licensee MDPI, Basel, Switzerland. This article is an open access article distributed under the terms and conditions of the Creative Commons Attribution (CC BY) license (https:// creativecommons.org/licenses/by/ $4.0 /)$.
Abstract: As microRNAs (miRNAs) are important expression regulators of coding RNA, it is important to characterize their role in the interaction between hosts and pathogens. To obtain a comprehensive understanding of the miRNA alternation in Bombyx mori (B. mori) infected with Nosema bombycis ( $N$. bombycis), RNA sequencing and stem-loop qPCR were conducted to screen and identify the significantly differentially expressed miRNAs (DEmiRNAs). A total of 17 such miRNAs were identified in response to $N$. bombycis infection, among which miR6498-5p efficiently inhibited the proliferation of $N$. bombycis in BmE-SWU1 (BmE) cells by downregulating pyridoxal phosphate phosphatase 2 (BMPLPP2). In addition, a fluorescence in situ hybridization (FISH) assay showed that miR6498-5p was located in the cytoplasm of BmE cells, while it was not found in the schizonts of $N$. bombycis. Further investigation of the effect of BmPLPP2 on the proliferation of schizonts found that the positive factor BmPLPP2 could facilitate N. bombycis completing its life cycle in cells by overexpression and RNAi of BmPLPP2. Our findings offer multiple new insights into the role of miRNAs in the interaction between hosts and microsporidia.

Keywords: B. mori; N. bombycis; microRNA; miR6498-5p; BmPLPP2; anti-microsporidian

\section{Introduction}

Microsporidia are obligate intracellular parasites that infect multiple species of invertebrates and vertebrates, including humans. These parasites can lead to huge economic losses to sericulture, cause colony mortality in honey bees, and cause health concerns in human patients with immunodeficiency [1,2]. Microsporidia have an extremely reduced genome ranging from 2.3 to $23 \mathrm{Mb}$, indicating an extreme dependence of the parasite on the host for biochemical processes $[3,4]$. There have been significant advances in the parasitological, evolutionary, and genomic research on microsporidia since multiple genome sequences were sequenced [5-13]. In addition, transcriptomics analysis of microsporidia infection in hosts has revealed a complicated and interconnected interaction in which microRNA (miRNA) as a key gene expression regulator is involved. However, the functions of most miRNAs in the host-microsporidia interaction remain unclear.

MiRNA are a class of small RNA molecules that were first found in Caenorhabditis. elegans by functional characterization of lin-4. This RNA can be processed into a $22 \mathrm{nt}$ single-stranded RNA, and it has a post-transcriptional regulatory function via incomplete complementary binding with the $3^{\prime} \mathrm{UTR}$ region of the target gene [14]. To date, more than 30,000 miRNAs have been found in multiple species, including animals, plants, and microbes. The functional characterization of miRNAs in host-pathogen interactions has been reported in the field of host-virus interactions. The host miRNAs can influence viral replication and pathogenesis by direct binding to the RNA virus genome or by causing 
alterations in the host transcriptome by targeting host genes $[15,16]$. MiRNA also plays an important role in regulating host internal homeostasis through miRNA-microbiota interactions [17]. Comparatively few studies have reported miRNA-mediated host-fungus interactions. Some plants are able to secrete miRNAs and transport them into the pathogen via an indistinct mechanism, thereby reducing the virulence of the pathogen and enhancing the resistance of the host [18]. As a fungus-like pathogen, a comprehensive investigation of miRNA-mediated regulation of microsporidia could provide insights into the hostmicrosporidia interaction.

As a model organism of Lepidoptera, silkworm is not only widely used in scientific research, but also one of the important economic animals in the world. N. bombycis infects silkworms and causes Pebrine, a chronic disease that results in significant damage to sericulture. As the first discovered microsporidian parasite, the N. bombycis genome has been sequenced [19]. As a parasite, N. bombycis is an ideal model for research on the host-microsporidia interaction due to the lack of infectivity to human beings and the ease of construction of an N. bombycis-infected cell model. The functional characterization of miRNA in the interaction between B. mori and N. bombycis could provide a reference for research into the mechanism of microsporidian infection and host defence and the regulatory role of key miRNAs in this interaction.

To elucidate the regulatory mechanism of miRNA in response to $N$. bombycis infection in B. mori, we identified the DEmiRNAs in N. bombycis infected BmE cells and explored the mechanism of DEmiRNAs in the regulation of N. bombycis proliferation. In this study, we identified 17 DEmiRNAs in infected BmE cells at $48 \mathrm{~h}$ by RNA sequencing and stem-loop qPCR. The $\beta$-tubulin of $N$. bombycis had a higher expression level at $48 \mathrm{~h}$ than at other time points, and immunofluorescence analysis suggested a rapid proliferation of schizonts at $48 \mathrm{~h}$. A highlighted miRNA, miRNA6498-5p, was further studied regarding its functional characterization and the mechanism of miR6498-5p inhibiting the proliferation of schizonts by downregulating BmPLPP2. Our results indicated a significant regulatory role of host miRNA in the defense against the pathogen.

\section{Materials and Methods}

\subsection{Sample Preparation}

BmE-SWU1 cells were maintained at $27^{\circ} \mathrm{C}$ in Grace Insect medium supplemented with $10 \%(v / v)$ fetal bovine serum (Gibco, Waltham, MA, USA). N. bombycis CQ1 strain was conserved in the China Veterinary Culture Collection Center (CVCC No. 102059). Mature spores were germinated in $0.1 \mathrm{M} \mathrm{KOH}$ solution and were then added to BmE cells for infection at a ratio of 10-30:1 Cell samples were collected and sent for RNA sequencing at $48 \mathrm{~h}$ after infection with $N$. bombycis.

\subsection{RNA Sequence and Data Analysis}

RNA sequence and data analysis were performed as described previously [20]. A certain range of length from clean reads was chosen for downstream analyses. The small RNA tags were mapped to $B$. mori reference sequences by Bowtie without mismatch, and the mapped small RNA tags were used to search for known miRNAs by using miRBase20.0 as a reference [21]. The available software programs miREvo andmirdeep2 were integrated to predict novel miRNAs through exploring the secondary structure, the Dicer cleavage site, and the minimum free energy of the small RNA tags unannotated in the former steps [22,23].

\subsection{Quantification of miRNA and Target Gene Prediction of miRNAs}

Quantification of miRNA expression levels was estimated as TPM (transcripts per million) [24]. Differential expression analysis of samples was performed using the DEGseq (2010) R package. The $p$-values were adjusted using qvalues [25]. A q value $<0.01$ and $\mid \log 2$ (fold change) $\mid>1$ were set as the threshold criteria for significantly differential expression by default; afterward, stem-loop qPCR was adopted to validate the expression 
levels of significantly differentially expressed miRNAs (DEmiRNAs). Prediction of the target genes of miRNAs was performed by miRanda-3.3a [26]. We used KOBAS software to statistically test the enrichment of the target gene candidates in KEGG pathways [27].

\subsection{Real-Time Quantitative Polymerase Chain Reaction ( $q P C R$ )}

Cell samples were treated with lysis buffer, and miRNA was separated from total RNA by using a MolPure ${ }^{\circledR}$ Cell/Tissue miRNA Kit (Yeasen, Shanghai, China). For qPCR, specific stem-loop primers were used for reverse transcription following the instructions of a PrimeScript ${ }^{\mathrm{TM}} \mathrm{RT}$ reagent kit with gDNA Eraser (Takara, Beijing, China), and qPCR reactions were performed in a CFX96 Real-Time System using NovoStar SYBR qPCR SuperMix plus (Jinan, China). The qPCR program was as follows: $95^{\circ} \mathrm{C}$ for $30 \mathrm{~s}, 40$ cycles of $95^{\circ} \mathrm{C}$ for $5 \mathrm{~s}$, and $60{ }^{\circ} \mathrm{C}$ for $30 \mathrm{~s}$. Data were analyzed using snRNA U6 and sw22934 (B. mori eukaryotic translation initiation factor $4 \mathrm{~A}$ ) as an endogenous control to quantify the expression levels of miRNA and mRNA, respectively, using the $2^{-\Delta \Delta \mathrm{Ct}}$ method [28]. Meanwhile, small subunit ribosomal RNA gene copies of $N$. bombycis were detected by qPCR to examine their proliferation in BmE cells.

\subsection{Vector Construction}

To explore the functions of DEmiRNAs, the U6 promoter of B. mori was used to initialize the expression in BmE cells. In addition, a heterogenous RNA with a length of $21 \mathrm{nt}$ was synthesized as a negative control (NC), and miRNA expression cassettes were cloned into pSL1180 plasmids and sequenced for use. Specific miRNA inhibitors were synthesized by the Tsingke Biotechnology Company (Beijing, China). The ORF of BmPLPP2 was cloned into a pIZV5/HIS vector and named pIZ-BmPLPP2. Related primers and RNA sequences are listed in the Supplementary Table S1.

\subsection{Fluorescence In Situ Hybridization (FISH)}

To clearly identify the location of miR6498-5p in BmE cells infected by N. bombycis at $48 \mathrm{~h}$, a digoxin-labeled specific probe was synthesized, and a miRNA fluorescence in situ hybridization (FISH) assay was conducted according to the instructions of a D-T-G type miRNA in situ hybrid kit. N. bombycis $\beta$-tubulin rabbit polyclonal antibody was used to locate the positions of schizonts after hybridizing the probe to the slide. All slides were examined under a super resolution laser Scanning confocal microscope with the proper filter (Olympus, Tokyo, Japan).

\subsection{Western Blotting}

Cell samples were lysed in RIPA lysis buffer (Biyuntian, Beijing, China) containing phenylmethanesulfonyl fluoride (PMSF) with a final concentration of $1 \mathrm{mM}$, and lysates were centrifuged at $12,000 \times \mathrm{g}$ for $10 \mathrm{~min}$ at $4{ }^{\circ} \mathrm{C}$. The supernatant was collected, and the concentration of total proteins was quantified by the BCA Protein Assay (Biyuntian, China) after centrifugation. Samples were separated by SDS-PAGE and transferred to a polyvinylidene fluoride (PVDF) membrane (Millipore, Middlesex County, MA, USA). The $\alpha$-tubulin of B. mori was used as an endogenous control to quantify the expression level of $\alpha$-PTP2 of $N$. bombycis. HRP-conjugated anti-rabbit IgG (1:5000) was used as a secondary antibody. The bands were visualized using a Clarity Western ECL Substrate kit (Biyuntian, China).

\subsection{Dual Luciferase Assay}

The binding sequence of miR6498-5p on BmPLPP2 mRNA was predicted, then cloned into pGL3-IE1-FLuc and named pGL3-WT-BmPLPP2-Luc. The binding site to seed sequences 1-6 of miR6498-5p were mutated to the complementary bases and then cloned into pGL3-IE1-FLuc and named pGL3-MUT-BmPLPP2-Luc. Renilla luciferase was used as an endogenous control to calibrate the activities of firefly luciferase expressed by pGL3-IE1-RLuc. BmE cells were co-transfected with FLuc reporter vector, miR6498-5p expression vector, 
and RLuc reporter vector at a ratio of $4: 4: 1$. The luciferase activities were detected by a Dual-Glo Luciferase Assay System (Promega, Madison, WI, USA) at $48 \mathrm{~h}$ post transfection, and the values of FLuc/RLuc were calculated.

\subsection{Statistical Analysis}

All data are shown as the mean \pm standard deviation from three independent experiments. All statistical analyses of the values were conducted using a two-tailed unpaired Student $t$-test. Values with ${ }^{*} p<0.05$ mean significant difference, ${ }^{* *} p<0.01$ represents very significant difference. All the statistical analyses were performed using GraphPad Prism 7.0 software.

\section{Results}

\subsection{Characterization of the Proliferation of N. bombycis in BmE Cells}

To prepare the infected BmE cells for RNA sequencing, we first investigated the proliferation of $N$. bombycis in BmE cells by qPCR and immunofluorescence. Two specific genes, $\beta$-tubulin and SWP5, were chosen for qPCR detection. The results showed that the structural protein gene $\beta$-tubulin had a relatively high expression level at $48 \mathrm{~h}$, while the spore wall protein gene SWP5 was significantly upregulated after $48 \mathrm{~h}$, indicating a proliferation period of $N$. bombycis in BmE cells at this time (Figure $1 \mathrm{~A}, \mathrm{~B}$ ). Immunofluorescence analysis indicated clear amplification of schizonts at $48 \mathrm{~h}$ (Figure $1 \mathrm{C}$ ). Based on the above results, the BmE cells infected by $N$. bombycis at $48 \mathrm{~h}$ and normal control cells were sent for RNA sequencing.
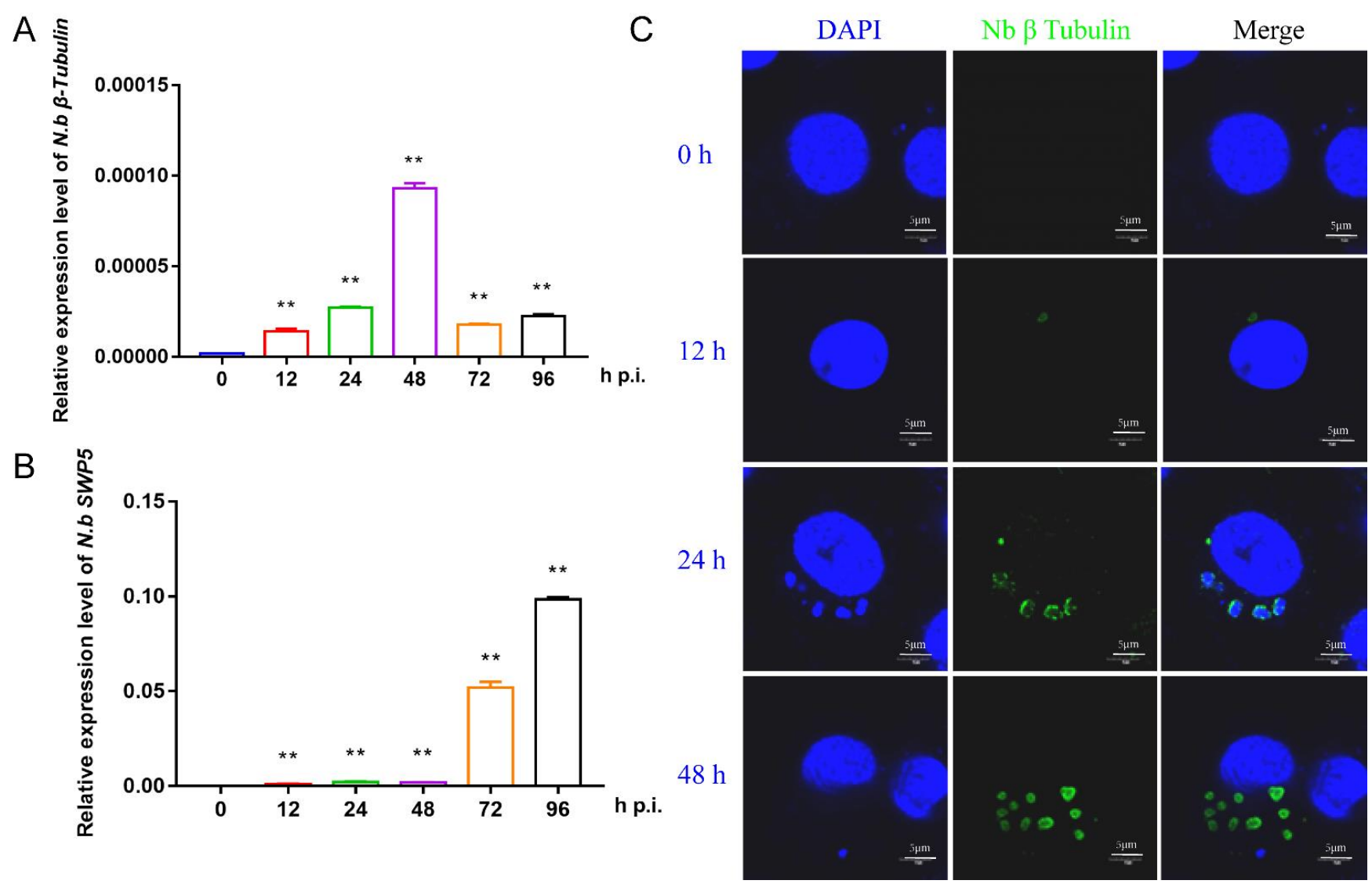

Figure 1. Characterization of the proliferation of $N$. bombycis in BmE cells (A) The expression level of the N. bombycis $\beta$-tubulin gene at different time points in BmE cells. (B) The expression level of N. bombycis swp5 at different time points in BmE cells. (C) Immunofluorescence analysis of schizont location in BmE cells at different time points. DAPI staining represents cell nuclei, and green fluorescence represents the $\beta$-tubulin protein of N. bombycis. All data represent the means of three replicates $\pm \mathrm{SD},{ }^{* *}, p<0.01$. 


\subsection{Data Analysis}

Data analysis was performed as described previously and the percentages of clean reads among raw reads of both groups were above 95\% [20]. In general, the sRNA length in animals ranges from 18 to $35 \mathrm{nt}$, so we chose the sRNA of this section to perform a statistical analysis of the length distribution (Figure 2A). The results showed that there was a clear difference in the distribution of each length of sRNA; the percentage of the sRNA length range from 18 to $26 \mathrm{nt}$ was increased in BmE cells infected by $N$. bombycis. In contrast, the proportion of sRNA with length range from 27 to $35 \mathrm{nt}$ was decreased relative to the uninfected group (Figure $2 \mathrm{~B}$ ). To obtain a comprehensive understanding of the alteration of sRNA, the sRNAs identified as having a length range from 18 to $35 \mathrm{nt}$ were mapped to B. mori reference sequences by Bowtie without mismatch. Totals of $16,960,665$ (Control group) and 9,023,874 (N.b group) sRNA reads with lengths from 18 to $35 \mathrm{nt}$ were obtained, of which $84.16 \%$ and $77.85 \%$, respectively, were mapped to reference sequences. To obtain a unique annotation for each sRNA, the sRNAs that were mapped to the reference sequences were annotated in the order of known miRNA $>$ rRNA $>$ tRNA $>$ snRNA $>$ snoRNA $>$ repeat $>$ gene $>$ novel miRNA. The results showed that the proportions of rRNA in both groups were lower than $37.5 \%$, indicating that our data were qualified for further analysis. The proportion of known miRNAs also had an increased tendency in cells that were infected by N. bombycis (Figure 2C,D). Our results showed that the sequence data were adequate for further analysis, and the host miRNAs reflected a response to infection by N. bombycis.

A

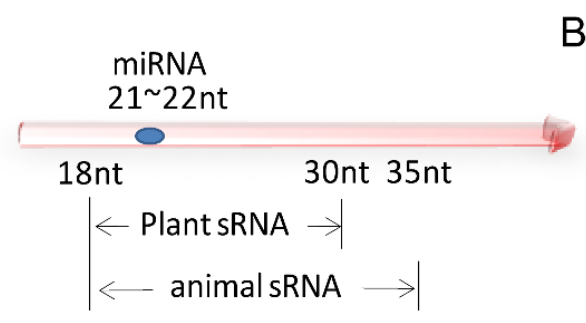

C

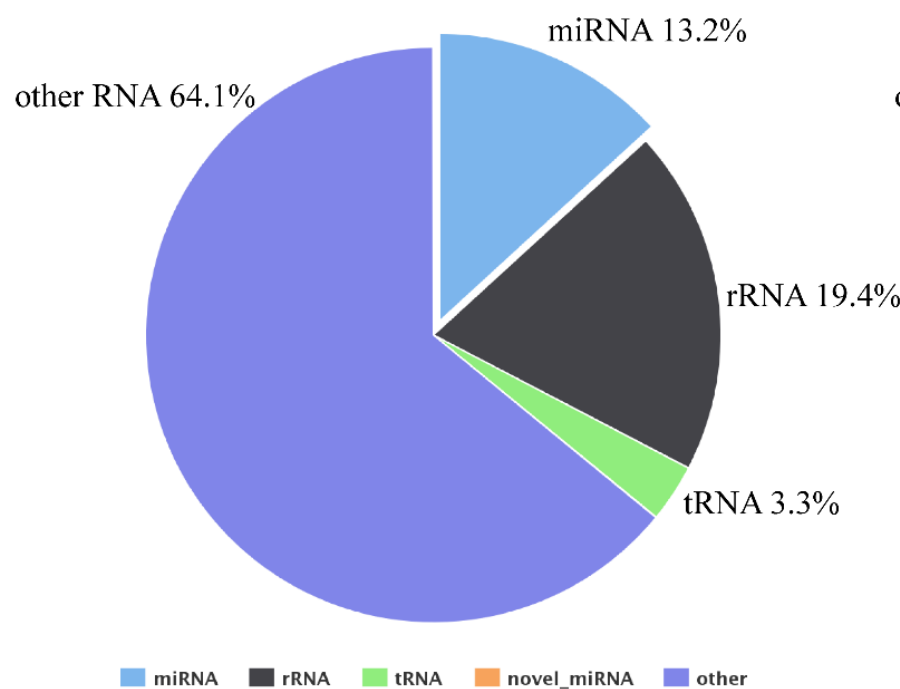

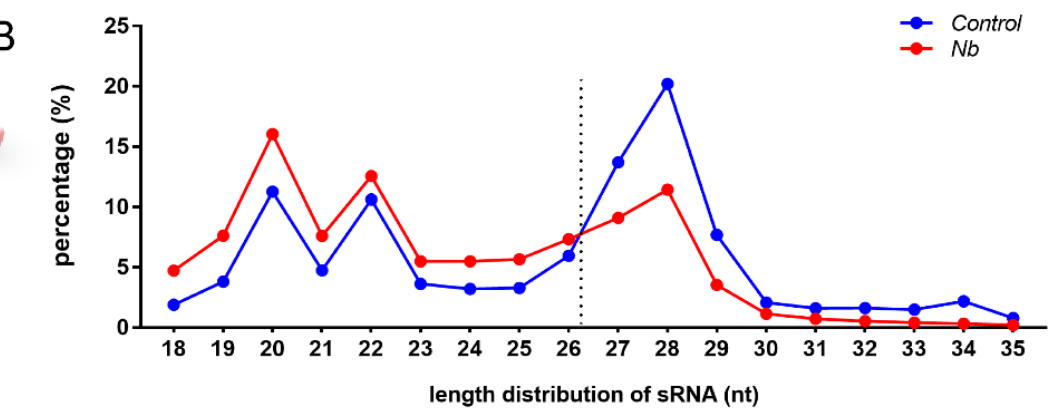

D

Figure 2. RNA sequence data analysis of BmE cells infected by N. bombycis and uninfected cells (A) Length range of sRNA in animals and plants. (B) Percentage of the sRNA mapped to the reference sequence of two groups. (C,D) The proportions of known miRNAs, rRNAs, tRNAs and other RNAs in mapped sRNA of the control group and the infected group. 


\subsection{Expression Analysis and Functional Detection of Host miRNAs}

To identify the miRNA response to infection in BmE cells, the expression levels of miRNAs were estimated as TPM (transcripts per million). A total of 33 miRNAs were differentially expressed with a q value $<0.01$ and $\mid \log 2$ (fold change) $\mid>1$, among which 11 miRNAs were upregulated and 22 were downregulated (Figure $3 \mathrm{~A}$ ). Following the identification, stem-loop qPCR was conducted to validate the expression levels of the 33 miRNAs. The results showed that 17 miRNAs were identified to have different expression with $\mid$ fold change $\mid>2$ (Figure 3B).

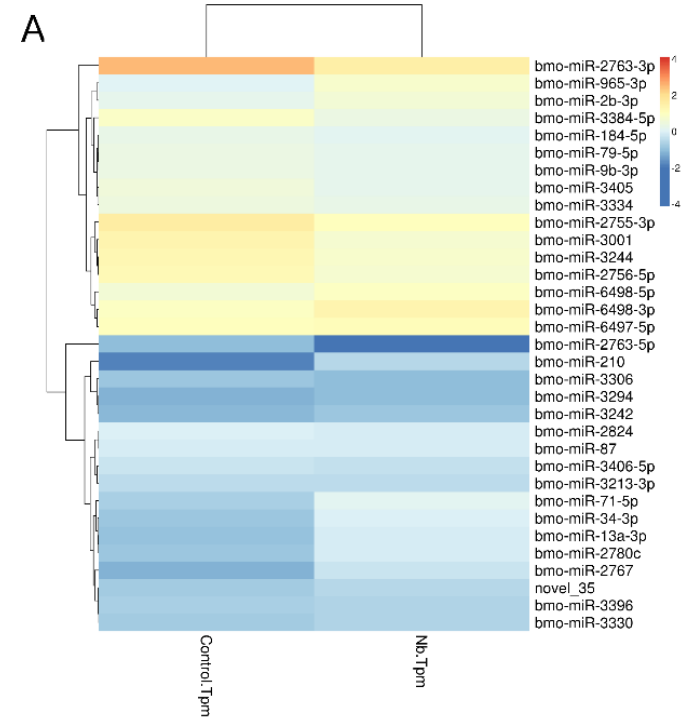

C

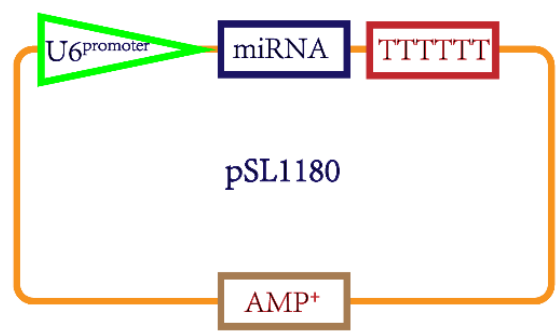

D

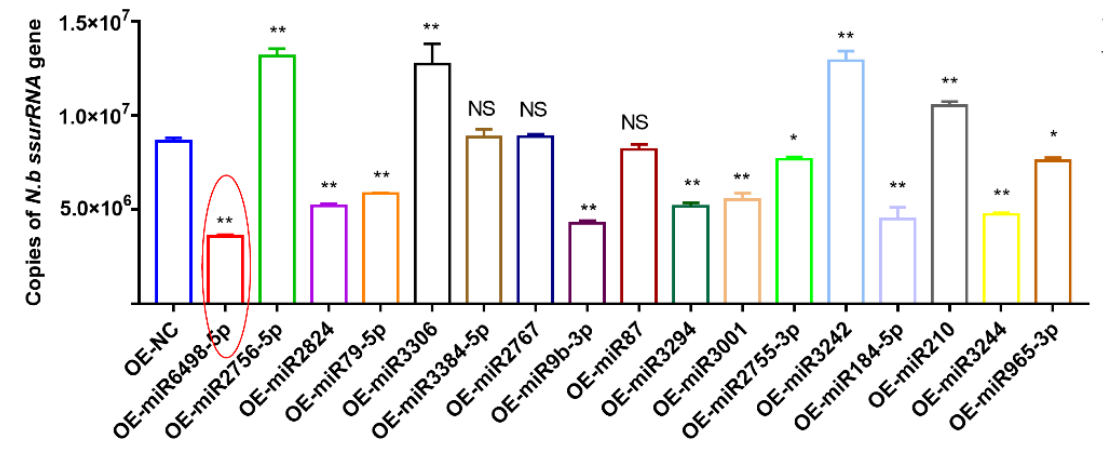

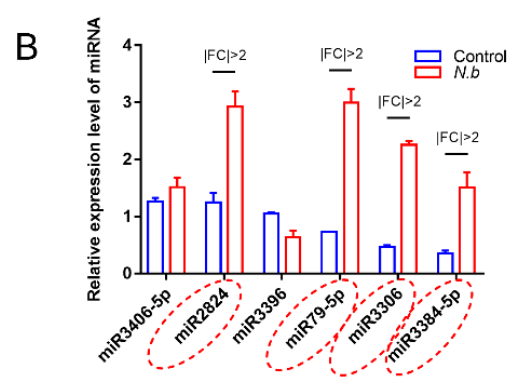
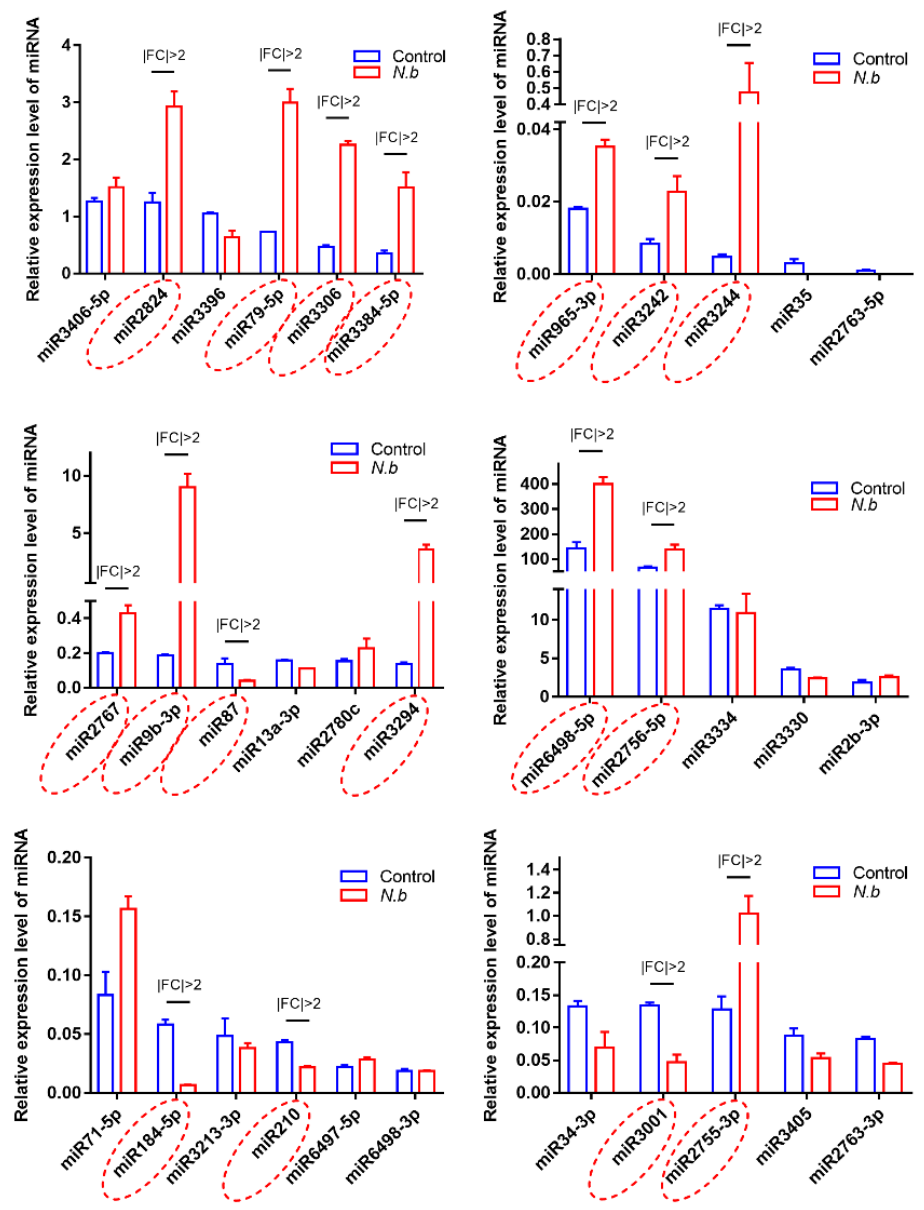

E

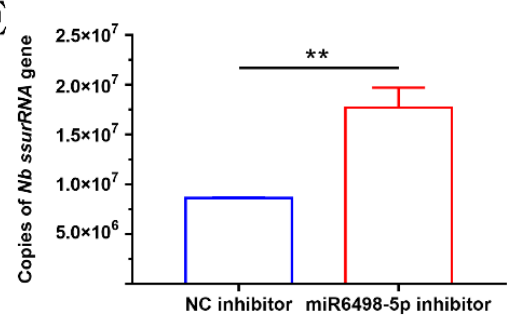

Figure 3. Validation of DEmiRNAs and the effect on N. bombycis proliferation (A) Heat map of DEmiRNAs screened by RNA sequence. (B) Stem-loop qPCR analysis of DEmiRNAs alteration after the infection with N. bombycis. (C) Vector construction for the expression of DEmiRNAs. (D) Copy analysis of N. bombycis ssurRNA gene in BmE cells transfected with different miRNA expression vectors by real-time quantitative polymerase chain reaction (qPCR) at $48 \mathrm{~h}$ post-infection. (E) Analysis of N. bombycis proliferation in BmE cells transfected with the inhibitor of miR6498-5p. All data represent the means of three replicates $\pm \mathrm{SD},{ }^{* *}, p<0.01, \mathrm{NS}, p>0.05$. 
To explore the functions of these 17 DEmiRNAs in N. bombycis proliferation, we constructed expression vectors for the 17 DEmiRNAs. The U6 promoter and TTTTTT were used for miRNA transcription and termination, respectively (Figure 3C). All vectors were sequenced prior to use. Then, BmE cells were infected with $N$. bombycis after being transfected with the DEmiRNA expression vector. The copy numbers of the $N$. bombycis small subunit rRNA (ssurRNA) gene in BmE cells were detected by qPCR at $48 \mathrm{~h}$ post infection, and the results showed that 14 DEmiRNAs had various effects on the proliferation of schizonts, among which four miRNAs had a stimulative effect on N. bombycis, while the others could inhibit the proliferation of schizonts in BmE cells. In particular, miR6498-5p displayed more effective inhibition of the replication of the ssurRNA gene (Figure 3D). In contrast to the inhibitory effect of miR6498-5p, the inhibitor of miR6498-5p significantly promoted the proliferation of schizonts (Figure 3E). Our results provide a comprehensive understanding of the response and regulation of host miRNA to $N$. bombycis infection.

\subsection{Functional Characterization and Location of miR6498-5p in BmE Cells}

MiR6498-5p is a sRNA with a length of $23 \mathrm{nt}$, and the precursor has a typical hairpin structure. The mature sequence of miR6498-5p is shown in Figure 4A. The qPCR analysis found that the expression pattern of miR6498-5p was similar to that of $N$. bombycis $\beta$ tubulin in infected cells, suggesting a close connection between the B. mori and N. bombycis (Figure $4 \mathrm{C}$ ). It has been reported that host miRNAs could be transported to pathogens and target the genes of the pathogens. To explore whether miR6498-5p could target the genes of N. bombycis, and then inhibit its proliferation, a FISH assay was conducted to confirm the location of miR6498-5p in infected cells. The results showed that miR6498-5p was located in the cytoplasm of BmE cells, with the same locations as 18s rRNA (Figure 4B). In the infected cells, miR6498-5p was present in the BmE cells but could not be found in the schizonts (Figure 4D), indicating that miR6498-5p inhibited the proliferation of N. bombycis by targeting the genes of BmE cells.

\subsection{Regulation of the Target Gene by miR6498-5p}

To identify the target of miR6489-5p, potential genes were predicted by miRanda-3.3a. A total of 271 genes were obtained with the criterion of minimum free energy $<-20 \mathrm{kcal} / \mathrm{mol}$. To obtain comprehensive knowledge concerning the functions of these target genes, KEGG enrichment analysis was performed by KOBAS (http://kobas.cbi.pku.edu.cn/ (25 December 2020)). The bubble diagram (Figure 5A) shows the top 23 KEGG enrichment pathways, among which the top nine pathways were involved in spliceosome, fatty acid biosynthesis, longevity regulating pathway, SNARE interactions in vesicular transport, amino sugar and nucleotide sugar metabolism, dorso-ventral axis formation, ubiquitin mediated proteolysis, terpenoid backbone biosynthesis, and vitamin $\mathrm{B} 6$ metabolism.

To find the genes that were regulated by miR6498-5p, we aimed to identify those genes that had a predicted binding site with miR6498-5p and could simultaneously be downregulated by N. bombycis infection. According to the transcriptome data, a total of 725 genes were significantly downregulated, among which 12 genes had a potential binding site with miR6498-5p (Figure 5B). To examine the regulation of miR6498-5p on the expression of the 12 genes, the expression levels were detected in BmE cells transfected with the expression vector for miR6489-5p. The results showed that two genes were downregulated with a fold change $>2$ relative to the control group (Figure 5 C). 
A

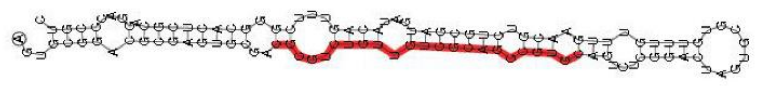

Mock

B
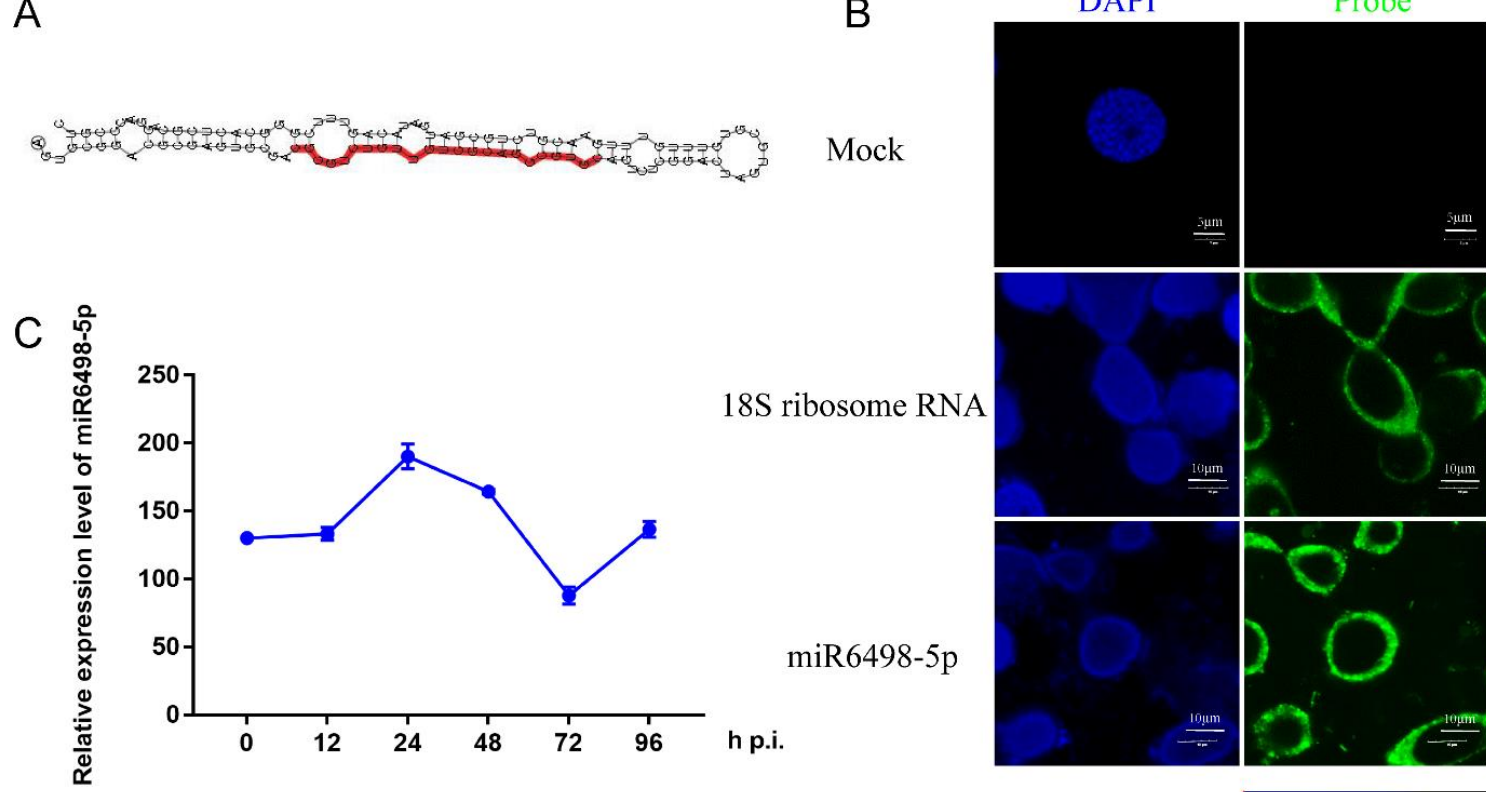

Probe

Merge

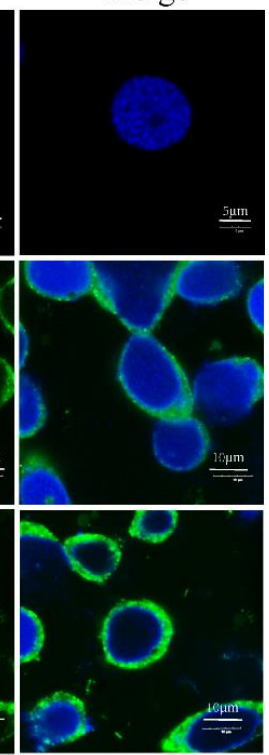

miR6498-5p
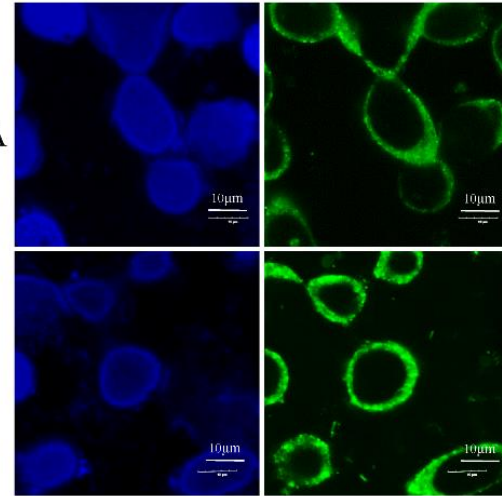

8 ribosome $\mathrm{RNA}$

DAPI

Probe of miR6498-5p

$\mathrm{Nb} \beta$ Tubulin

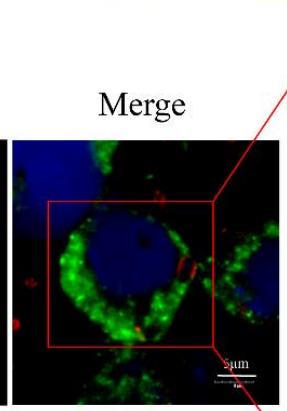

$\stackrel{5 \mu \mathrm{m}}{=\frac{15}{4}}$
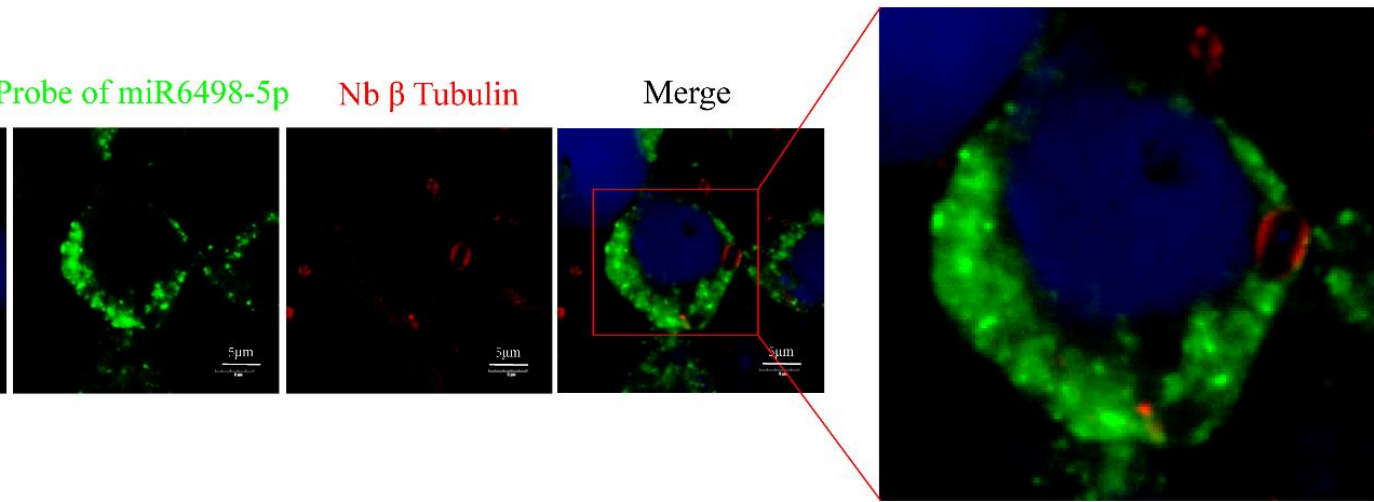

Figure 4. Characterization of miR6498-5p in BmE cells (A) Precursor structure of miR6498-5p. The red color represents the location and sequence of miR6498-5p. (B) Fluorescence in situ hybridization (FISH) of miR6498-5p and B. mori 18S RNA. The DAPI staining represents the nuclei and green fluorescence represents the location of miR6498-5p or 18S ribosomal RNA. (C) Alteration of the expression level of miR6498-5p with the infection of N. bombycis at different time points. (D) The location of miR6498-5p in BmE cells infected by N. bombycis. The green fluorescence represents the location of miR6498-5p, and the red fluorescence represents the locations of schizonts.

To validate that BmPLPP2 (BGIBMGA009579) was the target gene of miR6498-5p, RNA hybrid prediction and dual luciferase assays were performed. The results showed that the binding site of miR6498-5p was located in the ORF of BmPLPP2, and the seed sequence of the miRNA (1-9 nt) was completely complementary with the target sequence. The predicted binding structure of miR6498-5p with BmPLPP2 is shown in Figure 5D. The expression of miR6498-5p significantly decreased the expression of BmPLPP2 as shown by co-transfection of the expression vectors of miR6498-5p and BmPLPP2 in cells (Figure 5E). The dual luciferase assay showed that miR6489-5p decreased the value of FLuc/RLuc in the wild-type group, indicating negative regulation of miR6498-5p on BmPLPP2 (Figure 5F). Furthermore, a series of miR6498-5 $p$ mutants was constructed, and the regulatory effect of the mutants on BmPLPP2 expression disappeared (Figure 5G). Our results validated $B m P L P P 2$ as being the target gene of miR6498-5p. 
A

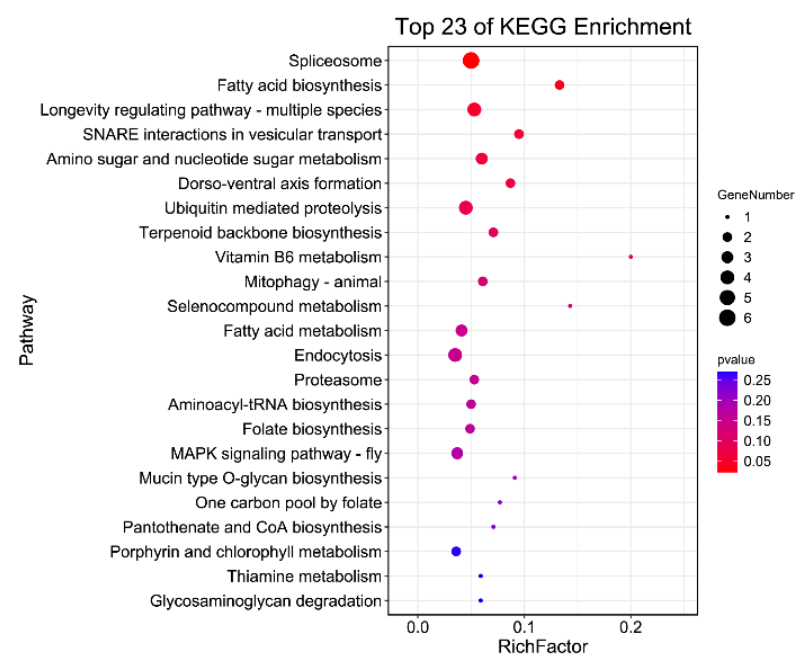

B down regulated gene

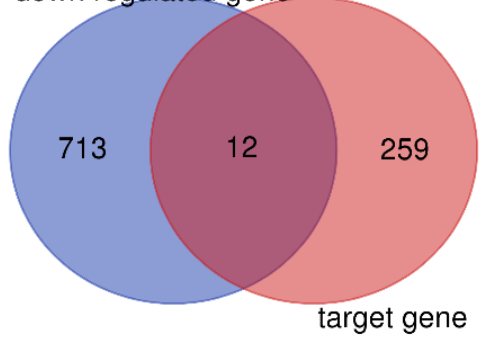

$\mathrm{F}$

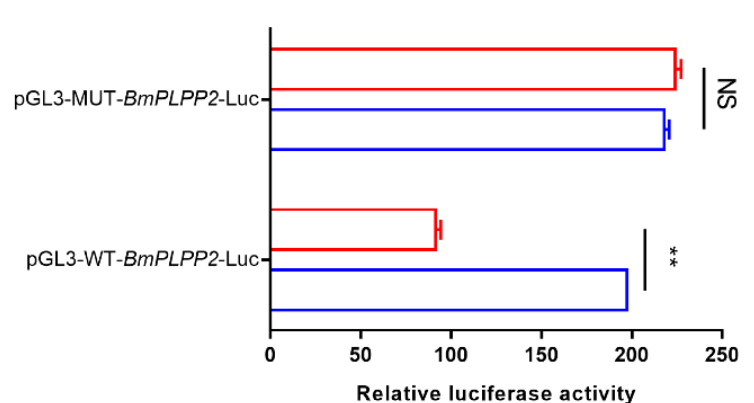

C

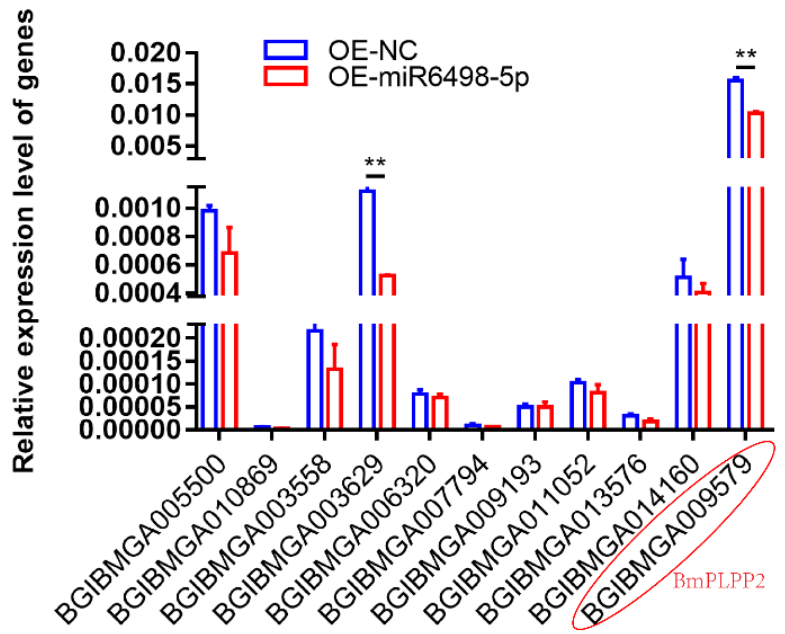

$\mathrm{E}$

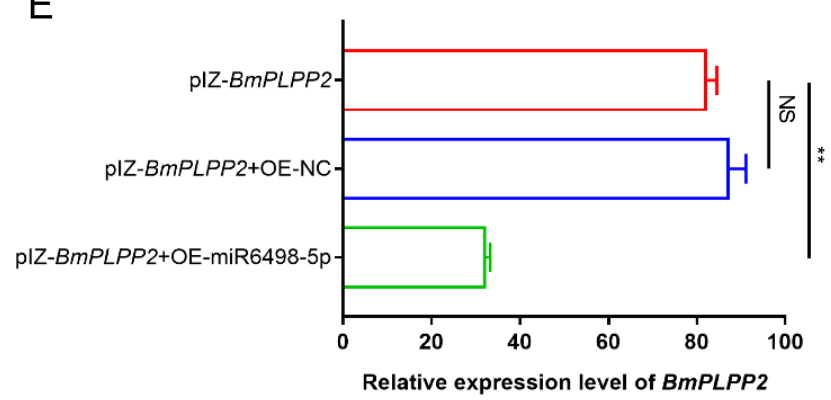

Figure 5. Identification of how miR6498-5p negatively regulates BmPLPP2 (A) KEGG enrichment analysis of the target genes of miR6498-5p predicted by miRanda-3.3a. (B) Venn diagram depicting the genes targeted by miR6498-5p and significantly downregulated by N. bombycis infection. (C) Expression of 12 genes obtained from the Venn diagram in BmE cells transfected with an OE-miR6498-5p vector. (D) The predicted binding structure of miR6498-5p with BmPLPP2 by RNA22. Green represents the miRNA, and red represents the mRNA. (E) qPCR analyzes the expression of BmPLPP2 in BmE cells by co-transfection with the expression vectors of BmPLPP2 and miR6498-5p (F) Dual luciferase assay analysis of the binding of miR6498-5p to the BmPLPP2. (G) qPCR analyzes the expression of BmPLPP2 in BmE cells by transfection with the mutants of miR6498-5p. Red sequences represent mutated bases. The dotted red line box represents the seed sequence of miR6498-5p. All data represent the means of three replicates $\pm \mathrm{SD},{ }^{* *}, p<0.01, \mathrm{NS}, p>0.05$. 


\subsection{BmPLPP2 Promotes Schizont Proliferation}

To investigate the effect of BmPLPP2 on N. bombycis proliferation, the pattern and location of BmPLPP2 expression were detected. The results showed that BmPLPP2 was located in the cytoplasm of $\mathrm{BmE}$ cells, and it was negatively expressed in relation to miR6489-5p (Figure 6A,B), strongly suggesting that BmPLPP2 was regulated by miR6498$5 \mathrm{p}$. Moreover, the ssurRNA gene copies and $\alpha$-PTP2 protein of N. bombycis were detected by qPCR and Western blotting in BmPLPP2 overexpressing cells. The results showed that $B m P L P P 2$ was significantly upregulated, and it significantly promoted the proliferation of schizonts (Figure 6C-E).

A

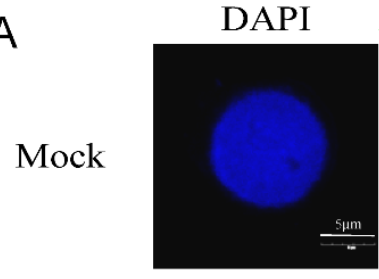

BmPLPP2

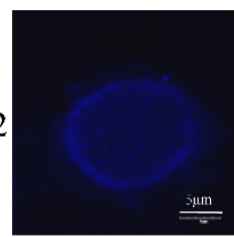

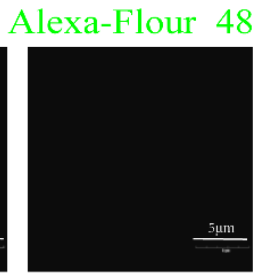

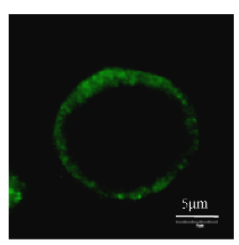

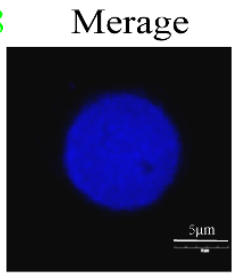

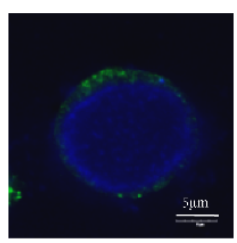

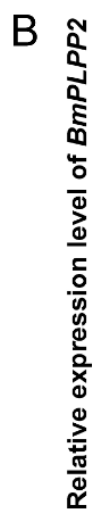

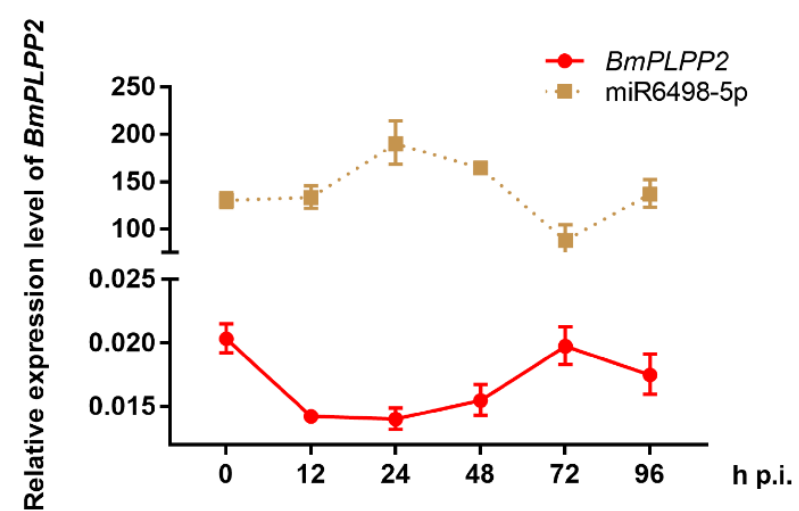

$\mathrm{E}$

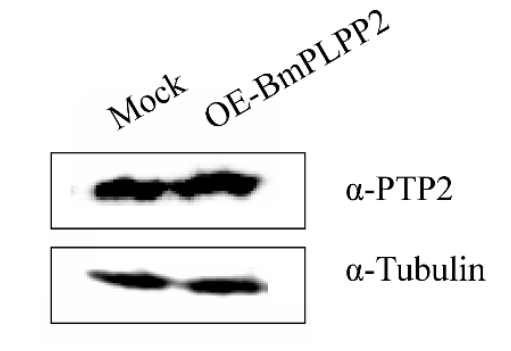

$\mathrm{H}$

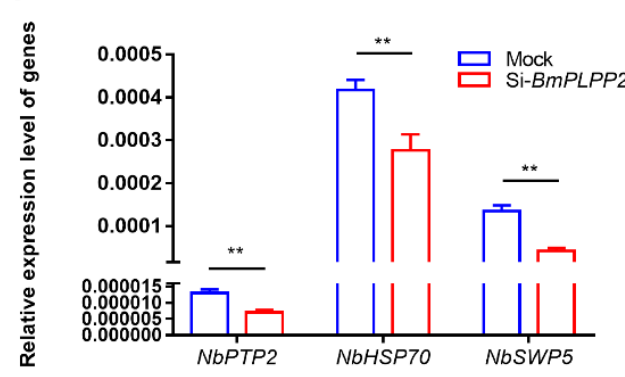

Figure 6. BmPLPP2 promotes the proliferation of $N$. bombycis (A) Expression alteration of BmPLPP2 after the infection of $N$. bombycis at different time points. (B) Immunofluorescence analysis of the location of BmPLPP2. DAPI staining represents the nuclei, and green fluorescence represents the location of BmPLPP2. (C) qPCR analyzes the expression of BmPLPP2 in BmE cells by transfected with pIZ-BmPLPP2. (D) Copy analysis of the N. bombycis ssurRNA gene in BmE cells transfected with pIZ-BmPLPP2. (E) Western blotting analysis of the expression of N. bombycis $\alpha$-PTP2 in BmPLPP2 overexpressing cells. (F) Expression analysis of BmPLPP2 in BmE cells transfected with siRNA. (G) Copy analysis of the N. bombycis ssurRNA gene in BmE cells by transfection with siRNA of BmPLPP2. (H) Gene expression analyses of N. bombycisPTP2, HSP70 and SWP5 in BmE cells by transfection with siRNA of BmPLPP2. All data represent the means of three replicates \pm SD, $* *, p<0.01$.

To further confirm the effect of BmPLPP2 on N. bombycis, an effective siRNA of BmPLPP2 was synthesized, and the interference efficiency was detected by qPCR (Figure 6F). The results showed that the ssurRNA gene copies were significantly decreased, and the 
expression levels of NbPTP2, NbHSP70 and NbSWP5 were inhibited in BmE cells by transfection with the siRNA of BmPLPP2 (Figure 6G,H). Taken together, the results suggest that miR6498-5p could inhibit $N$. bombycis proliferation by negatively regulating the expression of BmPLPP2.

\section{Discussion}

As a fungus-like parasite, microsporidia have become an increasingly important and fascinating model for the study of host-parasite molecular interactions since the discovery of $N$. bombycis in silkworms. At present, the molecular mechanism of the host-microsporidian interaction has been fully characterized, including the regulation of host metabolism, immune evasion, and host defense [29-33]. However, the regulatory mechanisms of miRNA in this relationship are poorly understood. The studies of the regulation of host miRNA on pathogens have great significance for the understanding of host-microsporidian interactions and for parasite research. In our study, 17 miRNAs were identified as having a response to the infection of $N$. bombycis, among which 14 miRNAs had a significant influence on the proliferation of schizonts. In addition, the mechanism of the inhibitory effect of miR6498-5p on N. bombycis was elucidated.

Since the discovery of lin-4, more than 30,000 miRNAs have been found in multiple species, including animals, plants, and microbes. MiRNA has a post-transcriptional regulatory function via incomplete complementary binding with the $3^{\prime} \mathrm{UTR}$ region of the target gene. Multiple important processes are regulated by miRNAs, including tumorigenesis, biological development, organ formation, and pathogen defense. Therefore, the functional characterization of miRNAs in their regulatory networks has received widespread attention. In host-virus interactions, the regulatory mechanisms of more than $30 \mathrm{miRNAs}$ in response to various viral infections have been identified. However, few miRNAs have been reported to mediate the interaction between the host and a fungus. Therefore, two specific genes, $N$. bombycis $\beta$-tubulin and spore wall protein 5 were detected in infected cells to identify RNA sequences present in a specific time period (Figure 1). The data showed that the proportion of host miRNA increased from $13.2 \%$ to $16.8 \%$ after the infection, and further validation found that 17 host miRNAs responded to infection by N. bombycis (Figures 2 and 3). Our results provide a comprehensive miRNA alteration reference for research concerning the host response to microsporidian infection.

In the interaction between host and pathogen, the host miRNAs can directly regulate the coding-RNA of pathogens. For example, cotton plants can secrete miR166, andmiR159 and transport these RNAs to Verticillium dahliae (a fungal pathogen), thereby enhancing the resistance of the host by downregulating the virulence related genes of the pathogen. Likewise, the pathogen miRNAs also could regulate the host messenger RNAs and thereby act as virulence factors during parasitism, for example in the parasitic plant Cuscuta campestris. In our research, $N$. bombycis acted as a fungus-like pathogen, and its coding RNA could not be regulated by silkworm miRNA according to the results of the FISH assay of miR6498-5p. Further study found that miR6498-5p could suppress the proliferation of $N$. bombycis by downregulating BmPLPP2, indicating indirect miRNA-mediated regulation by the host on microsporidia (Figure 4).

Due to the special regulation mechanism of miRNA on target genes, there are limits to identifying all the genes that are directly regulated by miRNA. This makes it a significant challenge to obtain a comprehensive functional characterization of miRNAs. In our research, a total of 271 target genes of miR6498-5p were predicted in B. mori. The KEGG enrichment analysis of these genes indicated involvement in multiple pathways, including spliceosome, fatty acid biosynthesis, and ubiquitin-mediated proteolysis. To identify the genes that were regulated by miR6498-5p in the interaction between B. mori and N. bombycis, we screened the genes that had a predicted binding site with miR6498-5p and could simultaneously be downregulated by N. bombycis infection. In this way, 12 genes were obtained, and the transcriptional regulation of miR6498-5p on these genes was detected. As a phosphatase, PLPP has an in vitro specificity to dephosphorylate pyridoxal $5^{\prime}$-phosphate (PLP) 
that acts as co-factor in more than 140 different enzyme reactions [34-36] and is especially involved in amino acid transport and metabolism. Multiple experiments demonstrated that miR6498-5p could target and negatively regulate BmPLPP2 and thereby suppress the proliferation of $N$. bombycis (Figures 5 and 6). The results indicated that the host may downregulate the mRNA level of BmPLPP2 by increasing the expression of miR6498-5p, thus altering the balance of amino acid metabolism to suppress the proliferation of N. bombycis (Figure 7). However, how the infection induces the upregulation of miR6498-5p is still unknown. In the upstream region of 20-hydroxyecdysone-responsive miR275 cluster, multiple ecdysone receptor elements have been found, which indicates an inducer-related induction mechanism of miRNA expression [37]. As an inhibitor, the induced upregulation of miR6498-5p may be due to the activation of host pathways involved in response to pathogen invasion. Our research provides an insight into the host response to pathogen infection and miRNA regulation during the host-microsporidian interaction.

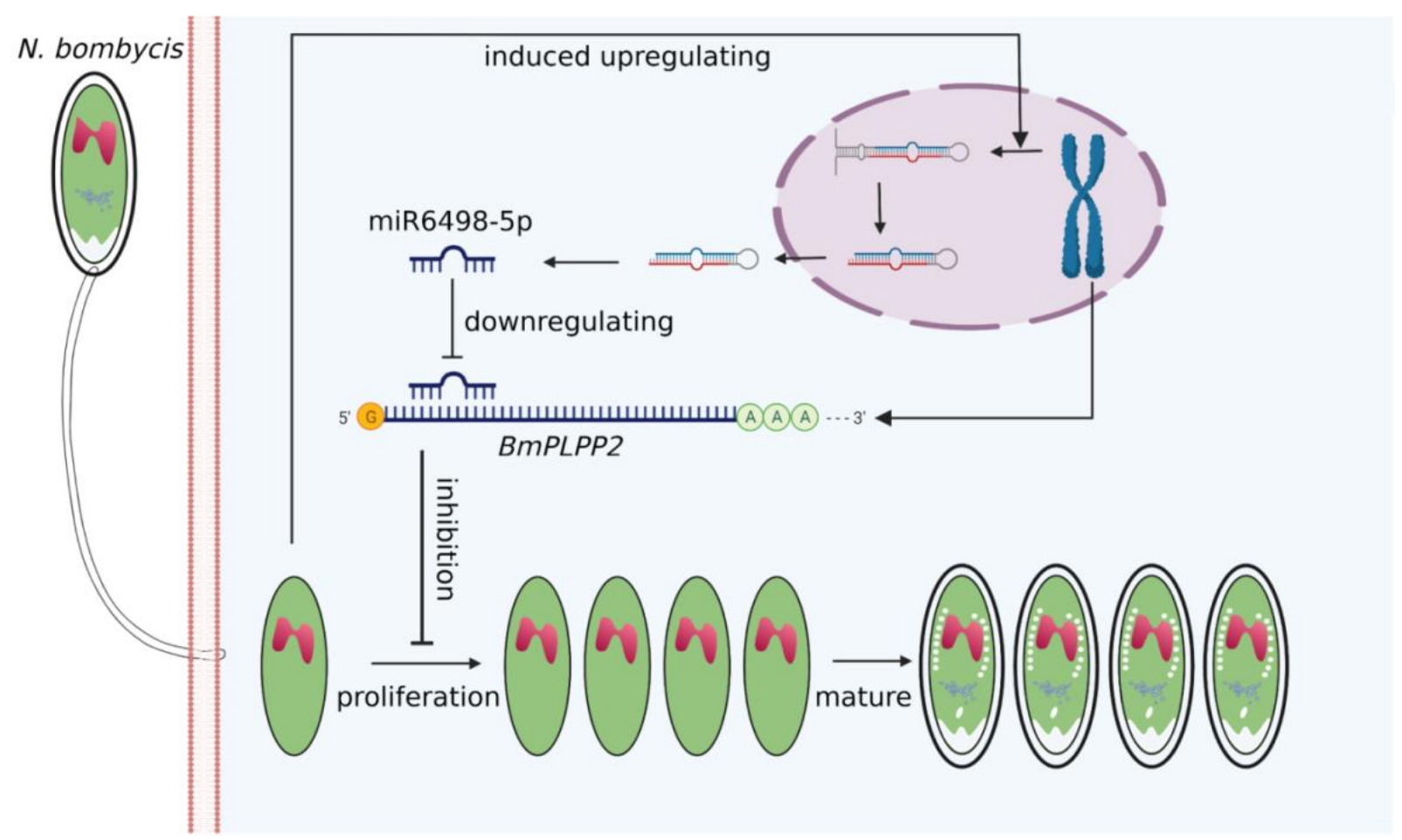

Figure 7. Model for host miR6498-5p inhibits N. bombycis proliferation by downregulating BmPLPP2.

\section{Conclusions}

This research identified a total of 17 silkworm miRNAs that response to N. bombycis infection, among which 14 miRNAs have significant effects on the proliferation of schizont. Meanwhile, the research also has demonstrated that miR6498-5p suppresses the proliferation of N. bombycis by downregulating BmPLPP2, an important factor in the regulation of host metabolism. The results have validated the important role of miRNA in the interaction between hosts and fungus-like parasites.

Supplementary Materials: The following are available online at https:/ / www.mdpi.com/article/10 .3390/jof7121051/s1, Table S1: Primers and related RNA sequence used in the study.

Author Contributions: Data curation, C.H. and Q.W.; Formal analysis, C.H.; Funding acquisition, M.P.; Investigation, C.H.; Methodology, B.D.; Project administration, Z.D. and M.P.; Resources, B.D.; Software, C.H. and Q.W.; Supervision, Z.D. and M.P.; Visualization, P.C.; Writing-original draft, C.H.; Writing-review \& editing, Z.D., P.C., C.L. and M.P. All authors have read and agreed to the published version of the manuscript. 
Funding: This study was funded by the National Natural Science Foundation of China (Grant Nos. 31872427 and 31902214), Natural Science Foundation of Chongqing (cstc2020jscx-msxmX0045 and cstc2019jcyj-msxmX0096), China Agriculture Research System of MOF and MARA and the Fundamental Research Funds for the Central Universities (SWU120008 and XDJK2020C010). We thank LetPub (www.letpub.com, accessed on 2 December 2021) for its linguistic assistance during the preparation of this manuscript.

Institutional Review Board Statement: This study did not involve humans or animals.

Informed Consent Statement: Not applicable.

Data Availability Statement: The RNA-seq data are available in NCBI with accession number: PRJNA760284.

Conflicts of Interest: The authors no potential conflict of interest.

\section{References}

1. Cox-Foster, D.L.; Conlan, S.; Holmes, E.C.; Palacios, G.; Evans, J.D.; Moran, N.A.; Quan, P.L.; Briese, T.; Hornig, M.; Geiser, D.M.; et al. A metagenomic survey of microbes in honey bee colony collapse disorder. Science 2007, 318, 283-287. [CrossRef] [PubMed]

2. Didier, E.S.; Didier, P.J.; Snowden, K.F.; Shadduck, J.A. Microsporidiosis in mammals. Microbes Infect. 2000, 2, 709-720. [CrossRef]

3. Han, B.; Weiss, L.M. Microsporidia: Obligate Intracellular Pathogens Within the Fungal Kingdom. Microbiol. Spectr. 2017, 5. [CrossRef] [PubMed]

4. Williams, B.A. Unique physiology of host-parasite interactions in microsporidia infections. Cell. Microbiol. 2009, 11, 1551-1560. [CrossRef] [PubMed]

5. Chen, L.; Gao, X.; Li, R.; Zhang, L.; Huang, R.; Wang, L.; Song, Y.; Xing, Z.; Liu, T.; Nie, X.; et al. Complete genome of a unicellular parasite (Antonospora locustae) and transcriptional interactions with its host locust. Microb. Genom. 2020, 6. [CrossRef] [PubMed]

6. Katinka, M.D.; Duprat, S.; Cornillot, E.; Metenier, G.; Thomarat, F.; Prensier, G.; Barbe, V.; Peyretaillade, E.; Brottier, P.; Wincker, P.; et al. Genome sequence and gene compaction of the eukaryote parasite Encephalitozoon cuniculi. Nature 2001, 414, 450-453. [CrossRef] [PubMed]

7. Keeling, P.J.; Fast, N.M. Microsporidia: Biology and evolution of highly reduced intracellular parasites. Annu. Rev. Microbiol. 2002, 56, 93-116. [CrossRef] [PubMed]

8. Keeling, P.J.; Fast, N.M.; Law, J.S.; Williams, B.A.; Slamovits, C.H. Comparative genomics of microsporidia. Folia Parasitol. 2005, 52, 8-14. [CrossRef]

9. Keeling, P.J.; McFadden, G.I. Origins of microsporidia. Trends Microbiol. 1998, 6, 19-23. [CrossRef]

10. Peters, M.J.; Suwannapong, G.; Pelin, A.; Corradi, N. Genetic and Genome Analyses Reveal Genetically Distinct Populations of the Bee Pathogen Nosema ceranae from Thailand. Microb. Ecol. 2019, 77, 877-889. [CrossRef]

11. Wadi, L.; Reinke, A.W. Evolution of microsporidia_An extremely successful group of eukaryotic intracellular parasites. PLoS Pathog. 2020, 16, e1008276. [CrossRef] [PubMed]

12. Delbac, F.; Peuvel, I.; Metenier, G.; Peyretaillade, E.; Vivares, C.P. Microsporidian invasion apparatus: Identification of a novel polar tube protein and evidence for clustering of $p t p 1$ and $p t p 2$ genes in three Encephalitozoon species. Infect. Immun. 2001, 69, 1016-1024. [CrossRef]

13. Han, B.; Polonais, V.; Sugi, T.; Yakubu, R.; Takvorian, P.M.; Cali, A.; Maier, K.; Long, M.; Levy, M.; Tanowitz, H.B.; et al. The role of microsporidian polar tube protein 4 (PTP4) in host cell infection. PLoS Pathog. 2017, 13, e1006341. [CrossRef]

14. Lee, R.C.; Feinbaum, R.L.; Ambros, V. The C. elegans heterochronic gene lin-4 encodes small RNAs with antisense complementarity to lin-14. Cell 1993, 75, 843-854. [CrossRef]

15. Trobaugh, D.W.; Klimstra, W.B. MicroRNA Regulation of RNA Virus Replication and Pathogenesis. Trends Mol. Med. 2017, 23, 80-93. [CrossRef] [PubMed]

16. Wong, R.R.; Abd-Aziz, N.; Affendi, S.; Poh, C.L. Role of microRNAs in antiviral responses to dengue infection. J. Biomed. Sci. 2020, 27, 4. [CrossRef] [PubMed]

17. Dong, J.; Tai, J.W.; Lu, L.F. miRNA-Microbiota Interaction in Gut Homeostasis and Colorectal Cancer. Trends Cancer 2019, 5, 666-669. [CrossRef]

18. Zhang, T.; Zhao, Y.-L.; Fang, Y.-Y.; Hua, C.-L.; Zhao, J.-H. Cotton plants export microRNAs to inhibit virulence gene expression in a fungal pathogen. Nat. Plants 2016, 2, 16153. [CrossRef] [PubMed]

19. Pan, G.; Xu, J.; Li, T.; Xia, Q.; Liu, S.L.; Zhang, G.; Li, S.; Li, C.; Liu, H.; Yang, L.; et al. Comparative genomics of parasitic silkworm microsporidia reveal an association between genome expansion and host adaptation. BMC Genom. 2013, 14, 186. [CrossRef]

20. Dong, Z.; Zheng, N.; Hu, C.; Deng, B.; Fang, W.; Wu, Q.; Chen, P.; Huang, X.; Gao, N.; Lu, C.; et al. Nosema bombycis microRNA-like RNA 8 (Nb-milR8) Increases Fungal Pathogenicity by Modulating BmPEX16 Gene Expression in Its Host, Bombyx mori. Microbiol. Spectr. 2021, 9, e0104821. [CrossRef] [PubMed]

21. Langmead, B.; Trapnell, C.; Pop, M.; Salzberg, S.L. Ultrafast and memory-efficient alignment of short DNA sequences to the human genome. Genome Biol. 2009, 10, R25. [CrossRef] 
22. Friedlander, M.R.; Mackowiak, S.D.; Li, N.; Chen, W.; Rajewsky, N. miRDeep2 accurately identifies known and hundreds of novel microRNA genes in seven animal clades. Nucleic Acids Res. 2012, 40, 37-52. [CrossRef]

23. Wen, M.; Shen, Y.; Shi, S.; Tang, T. miREvo: An integrative microRNA evolutionary analysis platform for next-generation sequencing experiments. BMC Bioinform. 2012, 13, 1-10. [CrossRef] [PubMed]

24. Zhang, G.; Gu, C.; Zhu, Y.; Luo, L.; Dong, D.; Wan, F.; Zhang, H.; Shi, G.; Sun, L.; Ye, D. ADIPOQ polymorphism rs182052 is associated with clear cell renal cell carcinoma. Cancer Sci. 2015, 106, 687-691. [CrossRef]

25. Storey, J.D. The positive false discovery rate: A Bayesian interpretation and the q-value. Ann. Stat. 2003, 31, 2013-2035. [CrossRef]

26. Enright, A.J.; John, B.; Gaul, U.; Tuschl, T.; Sander, C.; SMarks, D.S. MicroRNA targets in Drosophila. Genome Biol. 2003,5, R1. [CrossRef]

27. Mao, X.; Cai, T.; Olyarchuk, J.G.; Wei, L. Automated genome annotation and pathway identification using the KEGG Orthology (KO) as a controlled vocabulary. Bioinformatics 2005, 21, 3787-3793. [CrossRef] [PubMed]

28. Livak, K.J.; Schmittgen, T.D. Analysis of relative gene expression data using real-time quantitative PCR and the 2(-Delta Delta C(T)) Method. Methods 2001, 25, 402-408. [CrossRef]

29. Hua, X.; Xu, W.; Ma, S.; Xia, Q. STING-dependent autophagy suppresses Nosema bombycis infection in silkworms, Bombyx mori. Dev. Comp. Immunol. 2020, 115, 103862. [CrossRef]

30. Han, B.; Ma, Y.; Tu, V.; Tomita, T.; Mayoral, J.; Williams, T.; Horta, A.; Huang, H.; Weiss, L.M. Microsporidia Interact with Host Cell Mitochondria via Voltage-Dependent Anion Channels Using Sporoplasm Surface Protein 1. mBio 2019, 10, e01944-19. [CrossRef]

31. Huang, Y.; Zheng, S.; Mei, X.; Yu, B.; Sun, B.; Li, B.; Wei, J.; Chen, J.; Li, T.; Pan, G.; et al. A secretory hexokinase plays an active role in the proliferation of Nosema bombycis. PeerJ 2018, 6, e5658. [CrossRef]

32. Bao, J.; Liu, L.; An, Y.; Ran, M.; Ni, W.; Chen, J.; Wei, J.; Li, T.; Pan, G.; Zhou, Z. Nosema bombycis suppresses host hemolymph melanization through secreted serpin 6 inhibiting the prophenoloxidase activation cascade. J. Invertebr. Pathol. 2019, 168, 107260. [CrossRef] [PubMed]

33. Bakowski, M.A.; Desjardins, C.A.; Smelkinson, M.G.; Dunbar, T.L.; Lopez-Moyado, I.F.; Rifkin, S.A.; Cuomo, C.A.; Troemel, E.R. Ubiquitin-mediated response to microsporidia and virus infection in C. elegans. PLoS Pathog. 2014, 10, e1004200. [CrossRef] [PubMed]

34. di Salvo, M.L.; Contestabile, R.; Safo, M.K. Vitamin B(6) salvage enzymes: Mechanism, structure and regulation. Biochim. Et Biophys. Acta 2011, 1814, 1597-1608. [CrossRef]

35. Sugimoto, R.; Saito, N.; Shimada, T.; Tanaka, K. Identification of $Y b h A$ as the pyridoxal 5'-phosphate (PLP) phosphatase in Escherichia coli: Importance of PLP homeostasis on the bacterial growth. J. Gen. Appl. Microbiol. 2018, 63, 362-368. [CrossRef]

36. Huang, S.; Han, C.; Ma, Z.; Zhou, J.; Zhang, J.; Huang, L. Identification and characterization of a pyridoxal 5'-phosphate phosphatase in the silkworm (Bombyx mori). Comp. Biochem. Physiol. Part B Biochem. Mol. Biol. 2017, 205, 39-45. [CrossRef] [PubMed]

37. Jin, X.; Wu, X.; Zhou, L.; He, T.; Yin, Q.; Liu, S. 20-Hydroxyecdysone-responsive microRNAs of insects. RNA Biol. 2020, 17, 1454-1471. [CrossRef] [PubMed] 